\title{
¿Por qué tienen éxito y fracasan las personas con poder y sin poder? Poder y atribuciones de control ${ }^{*}$
}

\section{Why do People With and Without Power Succeed or Fail? Power and Control Attributions}

Recibido: enero 19 de 2009 ｜ Revisado: abril 2 de 2009 ｜ Aceptado: abril 16 de 2009

\author{
ROCÍO MARTÍNEZ-GUTIÉRREZ ** \\ ROSA RODRÍGUEZ-BAILÓN \\ Miguel MOYA
}

Universidad de Granada, Granada, España

Esta investigación ha sido realizada gracias a la financiación del proyecto de investigación financiado por El Ministerio de Educación y Ciencia de España (Ref SEJ2007/64362/PSI).

** Departamento de Psicología Social, Facultad de Psicología, Campus de Cartuja s/n, 18011 Granada. Correos electrónicos: mrocio@ugr.es; rrbailon@ ugr.es; mmoya@ugr.es

\section{RESUMEN}

El objetivo principal de la presente investigación es analizar el impacto del poder en las atribuciones causales; concretamente, se analizan las que realiza el observador ante el éxito y el fracaso de personas que difieren en el poder que poseen en su trabajo. Los resultados muestran que tanto los éxitos como los fracasos de las personas con alto poder, se explican mediante atribuciones controlables internas, específicamente, refiriéndose al esfuerzo de persona poderosa. Sin embargo, la explicación de los resultados logrados por personas con bajo poder presenta un patrón diferente. Mientras que el éxito se atribuye al esfuerzo del subordinado, el fracaso, a su poca habilidad. Los resultados se analizan en relación con hallazgos anteriores y a su implicación en el mantenimiento del statu quo.

Palabras clave autores

Atribución, observador, poder, estatus, control, habilidad, esfuerzo.

Palabras clave descriptores

Atribución, psicología social, prestigio ocupacional, poder, ciencias sociales.

\section{A B S T R A C T}

The main goal of this research is to analyze the impact of power in the causal attribution. More especifically, we analyze the attributions made by the observer about people's success and failure who differ in the power that they have in their workplace. Results show that both the success and failure obtained by people with high power are explained by controlable, internal attributions, specifically, referring to the effort made by the powerful person. However, the explanation of the outcomes achieved by people with low power show a different pattern. While the success is attributed to the subordinate's effort, the failure is attributed to his or her low skill. The results were analyzed in relation to previous findings and their involvement in maintaining the status quo.

Key words authors

Attribution, Observer, Power, Status, Control, Skill, Effort.

Key words plus

Attribution, Social Psychology, Occupational Prestige, Power. Social Sciences. 
Las personas pasamos parte de nuestro tiempo intentando explicar muchos de los sucesos que ocurren en nuestras vidas. Este proceso explicativo no se circunscribe únicamente a nosotros mismos, sino que en numerosas ocasiones actuamos como jueces tratando de buscar las causas del comportamiento de amigos, compañeros de trabajo, o personas que en ocasiones ni siquiera conocemos. El proceso atributivo es uno de los más ampliamente investigados en Psicología Social, y numerosos estudios han descrito los tipos de atribuciones que la gente hace, los procesos que explican cómo se crean las distintas atribuciones y los efectos que dichas explicaciones tienen (Gilbert \& Malone, 1995; Peterson \& Selegman, 1984; Trope, 1986). En relación con sus efectos, se ha demostrado que la forma en la que la gente hace atribuciones tiene consecuencias importantes en su bienestar personal (Buchanan \& Selegman, 1995). Así, aquellas personas que hacen atribuciones internas sobre eventos negativos (e.j., suspendí porque no soy inteligente) se ponen a sí mismos en riesgo de problemas psicológicos como ansiedad, depresión o estrés (Fincham \& Bradbury, 1992; Peterson, 2000a). De esta forma, podemos comprender la relevancia de dicho proceso cognitivo en la conducta humana ya que si las atribuciones realizadas resultan inadecuadas, las consecuencias psicológicas, emocionales y conductuales serán perjudiciales para el individuo que las realiza, afectando la motivación y desempeño futuro. Por otro lado, entre los factores que influyen en el proceso atributivo hay un grupo que hace referencia a las características de la persona percibida, como por ejemplo su edad, sexo, o raza (Steensma, Den Hartigh \& Lucardie, 1994). En el presente artículo, se examina la importancia de una característica que también puede influir en el proceso de atribución causal: el poder que los individuos ostentan.

\section{La importancia del estudio del poder y otras variables asociadas}

Dado que la jerarquía social es una de las formas más frecuentes de organización social (Tie- dens, Ellsworth \& Mesquita, 2000), las personas a menudo se esfuerzan en alcanzar las expectativas que tienen sobre su propia posición en la estructura social, intentando mejorarla y evitando descender a posiciones inferiores (Jones \& Pittman, 1982; King, 1995; Winter, 1973). En su afán por mejorar su situación, las personas suelen utilizar símbolos de estatus para mostrar a los demás la posición social que ocupan, especialmente si ésta es alta. Además el resto de individuos prestan atención a estos símbolos con el objetivo de inferir el poder y el estatus que tienen los demás. Las posesiones materiales, el sexo, la raza, la educación que tienen o el tipo de puesto de trabajo que ocupan las personas, son claves útiles de la capacidad que los otros tienen para influir en los demás. En este sentido, en muchas de las definiciones de poder y estatus subyace la idea de influencia (Dahl, 1957; Kipnis, 1976; Turner, 1991).

Además, hay algunos comportamientos observables que parecen implicar la posesión de poder, casi por definición. Prestar dinero, dar órdenes, repartir recompensas o adjudicar castigos (Fiske, 1993; Keltner, Gruenfueld \& Anderson, 2003) son acciones que se relacionan positivamente con el control de los recursos que las personas tienen (Levinger, 1959; Lippitt, Polansky \& Rosen, 1952). Según Anderson y Galinsky (2006) el poder es un tema que fascina debido al interés que despiertan en la gente los comportamientos y pensamientos de las personas que tienen prestigio y poder en la sociedad. Aunque, típicamente, los poderosos pueden ser vistos como frívolos, el interés en comprender y predecir el comportamiento de las personas que ostentan poder es a menudo importante y útil ya que, a nivel práctico, entender las mentes de aquellas personas que ocupan altos cargos, nos ayuda a conocer cómo las personas de las que dependemos toman las decisiones que afectan nuestras propias vidas. Desde el punto de vista de los propios poderosos, la investigación sobre la posesión de poder ha puesto de manifiesto cómo dicha variable afecta diferentes procesos psicológicos como la atención (Guinote, 2007), la estereotipia (Rodriguez-Bailon, Moya \& Yzerbyt, 2000), o la toma de decisiones (Anderson \& Ga- 
linsky, 2006), etc. Por su parte, Kelter, Gruenfeld y Anderson (2003) proponen la teoría de la "Aproximación-Evitación", que permite integrar todos los hallazgos anteriores, distinguiendo entre el patrón de comportamiento que desarrollan los poderosos y los no poderosos. De acuerdo con su propuesta, la ostentación de poder se encuentra asociada con las recompensas y la libertad, por lo que activa tendencias comportamentales de "aproximación" o acercamiento. Por otra parte, la subordinación a otros se asocia con las amenazas y la constricción social y, por tanto, activa un patrón comportamental de evitación. En la misma linea, otra variable sociestructural típicamente relacionada con el poder, el estatus, también tiene repercusiones importantes en el comportamiento de los individuos. Investigaciones en distintos ámbitos muestran que tiene implicaciones en la identificación grupal (Ellemers, 1993), el nivel de satisfacción personal (Ellemers, Van Knippenberg \& Wilke, 1990), la autoestima (Cartwright, 1950), la amenaza a la identidad social de los individuos (Ellemers, Spears \& Doosje 1997), la implicación para mejorar el grupo, entre otras.

De forma resumida y de acuerdo con la propuesta de Keltner et al. (2003), podemos afirmar que las personas con poder se comportan de forma diferente a la gente que ocupa posiciones subordinadas. Probablemente, estos comportamientos a su vez afectan las expectativas que el observador tiene sobre el comportamiento prescriptivo de las personas con y sin poder. Así, por ejemplo, diversas investigaciones sugieren que la gente tiende a creer que las personas con alto estatus son más competentes, inteligentes e incluso más atractivos/ as que las personas que tienen bajo estatus (Darley, 1983; Georgesen \& Harris, 1998). Igualmente Fiske, Cuddy, Glick y Xu (2002) proponen que existen estereotipos generalizados de las personas en función del poder del que disfrutan. De esta forma, las personas poderosas son percibidas como competentes pero poco sociables y las personas no poderosas como carentes de dicha competencia pero más sociables.

\section{Atribuciones y poder}

El objetivo principal de la presente investigación es analizar el impacto del poder en las atribuciones que los perceptores hacen sobre diversos eventos que tienen lugar en el ámbito laboral. Overbeck, Tiedens y Brion (2006) demostraron que el observador tiende a hacer atribuciones disposicionales sobre las acciones de las personas que poseen poder, mientras que cuando se trata de personas con bajo poder aparece la tendencia contraria, esto es, los observadores tienden a hacer atribuciones situacionales. Quizás una de las razones por las que la gente poderosa da la impresión de ser afortunada es porque parecen libres de hacer cualquier cosa que deseen. Sin embargo, la gente que no tiene poder, puede dar la idea de estar sujetos a las limitaciones y al control de los demás o de las situaciones en las que se encuentran insertos. Como resultado, los observadores interpretan de diferente forma el comportamiento de las personas en función del poder que poseen. Si se trata de alguien poderoso, el perceptor entiende que actúa de cierta forma por su propia voluntad, mientras que si se trata de una persona sin poder, el observador interpreta que lo hace porque alguien se lo ordena o la situación lo obliga a ello. En el presente estudio, se consideró además interesante analizar dos casos concretos que suceden con relativa frecuencia dentro del ámbito laboral, la consecución de éxitos y fracasos. Según Weiner (1986), en función del tipo de explicaciones que las personas dan sobre el éxito o el fracaso del comportamiento de los demás y de sí mismos, así también varían los sentimientos asociados a los resultados obtenidos y, a su vez, éstos influyen en el tipo de conducta que las personas llevan a cabo con respecto a sí mismas y a los demás. De forma específica, el presente artículo se centra en dos tipos de causas: la habilidad y el esfuerzo. De acuerdo a la clasificación de Weiner (1986) sobre las dimensiones por las que se puede categorizar las causas implicadas en el proceso atributivo (locus, controlabilidad, etc.), la habilidad se considera interna e incontrolable, mientras que el esfuerzo sería también una causa interna pero controlable. Precisamente, bajo nuestro punto de vista, la dimensión 
"controlabilidad" cobra una importancia especial cuando relacionamos atribuciones con variables socio-estructurales como el poder. Debido a que el poder puede definirse como el control relativo que un individuo o grupo tiene sobre los resultados que los demás y él mismo pueden obtener (Fiske \& Dépret, 1996; Georgesen \& Harris, 1998), la dimensión de controlabilidad es especialmente relevante en el estudio de las atribuciones que se realizan tanto desde el punto de vista del observador como desde el punto de vista del actor. En el plano grupal, Lee, Peterson y Tiedens (2004) demuestran que cuando las empresas hacen atribuciones controlables sobre los resultados negativos obtenidos en el seno de la organización, se perciben como empresas con mayor control, incrementan sus ventas, y crean una imagen positiva de cara al observador. Es por ello que el objetivo principal del presente estudio, es analizar las explicaciones que las personas dan como observadoras del éxito o el fracaso de personas que se diferencian en el puesto de poder que ocupan dentro del ámbito laboral. Dado que las personas tienden a percibir a los poderosos como más competentes y más libres en las acciones que realizan, y por tanto con más control sobre sus resultados, y por el contrario a los subordinados como incompetentes y controlados por los demás o por factores externos a ellos, se plantean las siguientes hipótesis:

Hipótesis 1: El observador tenderá a explicar tanto el éxito como el fracaso del poderoso mediante atribuciones controlables (esfuerzo) en mayor medida que incontrolables (habilidad). Así, se considera que la tendencia a hacer atribuciones controlables se verá afectada por la posición de poder que ocupe el individuo. En este sentido, el observador hará más atribuciones controlables cuando el protagonista del escenario sea una persona poderosa. Se parte de que la percepción de las personas con poder como individuos que eligen hacer aquellas cosas que desean y que no están sometidos al control de superiores, llevará al observador a explicar tanto el éxito como el fracaso del poderoso, recurriendo a atribuciones controlables.
Hipótesis 2: El observador explicará de forma diferente el éxito y el fracaso de las personas que ocupan puestos de bajo poder en un contexto laboral. Así, el éxito se explicará mediante atribuciones controlables (esfuerzo) mientras que el fracaso se atribuirá a causas incontrolables (habilidad). En este caso, se hipotetiza que, debido a que uno de los rasgos estereotípicos de las personas con bajo poder es la incompetencia, éste influirá en la explicación que el observador haga sobre el éxito y el fracaso que dichas personas obtienen. Así, cuando el protagonista del escenario de bajo poder alcance un éxito, el perceptor tenderá a explicarlo mediante atribuciones controlables, esto es, por el esfuerzo que hace dicha persona por conseguir el logro de su objetivo debido a las pocas posibilidades (personales y situacionales) que tiene para alcanzarlo. Sin embargo, el mismo estereotipo sobre la incompetencia de los subordinados, hará que el observador explique el fracaso de los mismos mediante atribuciones incontrolables internas, es decir, mediante la baja habilidad que el subordinado posee para cumplir sus metas.

\section{Método}

\section{Participantes}

Ciento cuarenta y dos estudiantes de primer curso de la Facultad de Psicología de la Universidad de Granada participaron en el estudio. De ellos, 25 (17.6\%) fueron hombres y los 117 (82.4 \%) restantes mujeres. La media de edad fue de 19,03 años (DT $=4.13)$.

\section{Medidas y procedimiento}

Se elaboró un cuestionario formado por 4 escenarios en los que se describía unas situaciones enmarcadas en el contexto laboral. Para controlar un posible efecto de la variable sexo de los protagonistas, éste siempre fue femenino en los 4 escenarios. Dos de los escenarios hacían referencia a puestos de trabajo de alto poder (directora de un centro de Educación Especial y profesora de una escuela muy prestigiosa de Arte), siendo los 2 
episodios restantes, puestos de trabajo de bajo poder (empleada en una fábrica textil y empleada del Servicio de Limpieza). La manipulación del poder, además del tipo de trabajo propiamente dicho, se llevó a cabo indicando si la persona tenía capacidad de influencia en su entorno laboral o si por el contrario su papel decisorio era nulo. El siguiente ejemplo corresponde a una situación en la que el poder fue alto:

Ana Álvarez es directora de un Centro muy conocido de Educación Especial. Normalmente, la mayor parte de las decisiones que se toman en la Junta Directiva son decididas por ella ya que es una persona con bastante peso en el Centro.

De forma opuesta, el siguiente episodio describe un escenario de una persona de bajo poder:

Maribel Collantes trabaja como limpiadora en una empresa. Maribel sabe que ella no puede hacer sugerencias sobre la forma de planificar sus tareas ya que normalmente es la jefa del Servicio de Limpieza la que le organiza su trabajo.

El resultado que obtenía el protagonista del escenario en su lugar de trabajo también fue manipulado. Así, en la mitad de las situaciones el actor obtenía un éxito laboralmente hablando (p. ej., aumento de sueldo), mientras la otra mitad obtenía un fracaso relacionado con su trabajo (por ejemplo, no renovaba su contrato con la empresa).

Después de presentarles cada escenario se pedía a los participantes que estimaran la probabilidad (escala de 0 a 9 ) de cada una de las cuatro posibles causas que se detallaban como responsables del éxito o fracaso del protagonista del mismo. Con objeto de analizar el efecto de la controlabilidad de las atribuciones, uno de los objetivos prioritarios de la presente investigación, y dado que el locus de causalidad de las atribuciones presentadas fue siempre interno, se variaron las atribuciones solamente en la dimensión de controlabilidad. Además, debido a que una de las hipótesis contempla la importancia de "esfuerzo" y "habilidad" en las atribuciones que se realizan sobre las personas con bajo poder, se consideró interesante estudiar la preferencia de los participantes por este tipo de causas, teniendo presente que una de las dimensiones que las diferencia, es el grado de control que el protagonista tiene sobre las mismas.

Todos los participantes contestaron cada uno de los cuatro escenarios que formaban el cuadernillo que se les entregó al iniciar la sesión experimental. El orden de los escenarios fue contrabalanceado, para controlar su posible efecto.

\section{Resultados}

Para conocer las diferentes atribuciones que el observador hace sobre el éxito o fracaso de una persona con diferente posición de poder en su puesto de trabajo, se llevó a cabo un ANOVA con tres factores manipulados intraparticipantes: 2 Poder (alto vs. bajo) x 2 Resultado (éxito vs. fracaso) x 2 (Tipo de atribución: esfuerzo vs. habilidad).

El análisis mostró un efecto principal de la variable Poder, $F(1,139)=5.08, p=0.026)$; del Tipo de atribución, $F(1,139)=45.81, p=0.000)$; y del Resultado $(\mathrm{F}(1,139)=163.29, \mathrm{p}=0.000)$.

Pero de forma más interesante, y de acuerdo con las predicciones, estos efectos principales se vieron modulados por algunas interacciones de segundo y tercer orden. Así, la interacción que agrupa a las tres variables manipuladas: Poder X Resultado X Tipo de atribución también fue significativa, $F(1,139)=34.44, p<0.001$. Tal como se hipotetizó, se encuentran diferencias significativas en las atribuciones que el observador realiza sobre el éxito y el fracaso de las personas que ocupan puestos de bajo poder. Así, como puede verse en la Figura 1, los observadores eligen más causas controlables (esfuerzo) que incontrolables (habilidad) $(\mathrm{M}=6.86, \mathrm{DT}=1.55$ y $\mathrm{M}=6.18, \mathrm{DT}=1.91$ respectivamente para las causas controlables e incontrolables), para explicar el éxito que obtiene el protagonista en su puesto de trabajo en el escenario presentado, $t(140)=9.87, p<0.01$.

Por el contrario, cuando los participantes tienen que explicar el fracaso de las personas que ocupan puestos de bajo poder, recurren en mayor medida 


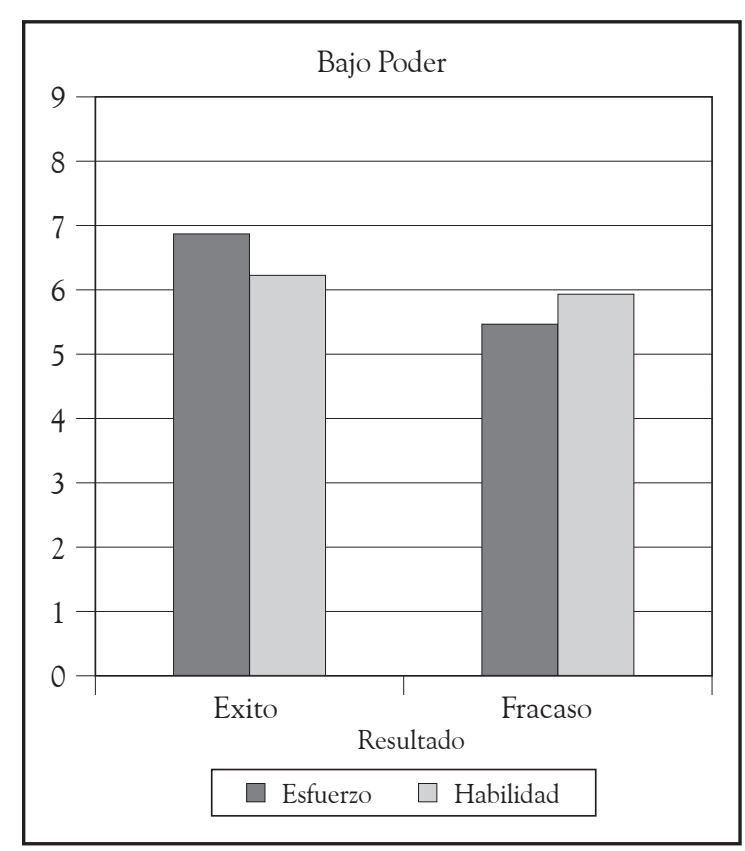

\section{FigurA 1}

Atribuciones del éxito y del fracaso que observadores hacen de personas que se encuentran en puestos de trabajo de bajo poder.

Fuente: elaboración propia.

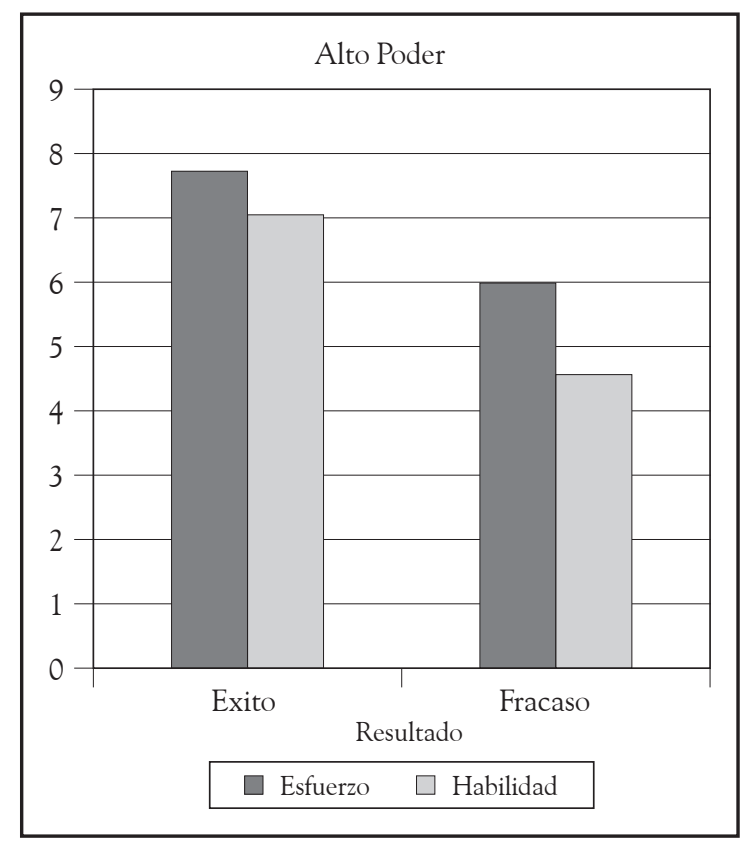

FiguRA 2

Atribuciones del éxito y del fracaso que observadores hacen de personas que se encuentran en puestos de alto poder.

Fuente: elaboración propia. a las causas incontrolables internas $(\mathrm{M}=5.93$, $D T=1.94)$ que a las controlables internas $(\mathrm{M}=$ $5.42, D T=1.90), t(141)=-2.61 ; p<0.05$.

Por otra parte, y en relación con las atribuciones que el observador hace sobre el resultado obtenido por personas que ocupan puestos de alto poder, tal como puede verse en la Figura 2, tanto cuando se trata de un éxito (Mcont $=7.68, D T=0.92$. Mincont $=7.02, D T=1.19)$, como de un fracaso $($ Mcont $=5,99, D T=1.66$. Mincont $=4.55, D T$ $=1.55)$ son las causas controlables las escogidas en mayor medida que las causas incontrolables internas, $t(141)=-7.41, p<0.001 ; y t(140)=$ $8.04 ; p<0.001$, para el éxito y el fracaso, respectivamente.

\section{Discusión}

Los resultados obtenidos en este estudio aportan conocimientos novedosos en el análisis de los efectos que tiene el poder y su naturaleza en las atribuciones causales. Con este objetivo, se pidió a los participantes que actuaran como observadores y que explicaran las causas de cuatro escenarios diferentes. Todos los escenarios hacían referencia a situaciones que ocurren de forma frecuente en el ámbito organizacional. Se manipuló el poder del puesto de trabajo que ocupaba el protagonista del episodio, creando dos escenarios en los que esta persona tenía puestos de responsabilidad en la empresa en la que trabajaba, y dos escenarios en los que el puesto de trabajo se caracterizaba por un nivel muy bajo de poder por parte del empleado que ocupaba dicho cargo. Además, se manipuló el resultado que dicha persona obtenía en su área de trabajo, enfatizando el éxito por un lado y el fracaso por otro. De esta forma, los participantes de la investigación debían valorar la importancia de dos tipos de causas concretas. De forma específica, el interés era conocer la importancia que la "controlabilidad" de las causas tiene en las atribuciones que las personas hacen sobre los eventos que les suceden a personas poderosas y no poderosas. La controlabilidad de las causas hace referencia a la capacidad del actor para influir en el resultado 
obtenido, así las causas controlables están sujetas a control volitivo mientras que las incontrolables no están sometidas a ningún tipo de control por parte del actor. Es por ello que se presentan dos tipos concretos de causas que coincidían en el locus de causalidad (ambas eran internas) pero que diferían en controlabilidad. De esta forma, se mostraron dos posibles causas explicativas del resultado obtenido por el actor: el esfuerzo (controlable, interna) y la habilidad (incontrolable, interna).

Además, el esfuerzo y la habilidad son dos causas con implicaciones relevantes en el estudio del poder. Según Weiner (1985) en función del tipo de explicaciones que las personas dan del éxito o del fracaso del comportamiento de los demás y de sí mismos, así varían los sentimientos asociados a los resultados obtenidos y, a su vez, éstos influyen en el tipo de conducta que las personas llevan a cabo. En esta línea, se puede pensar que no es lo mismo atribuir el fracaso de una persona a su poca capacidad o a su poco esfuerzo, ya que centrarse en la poca capacidad es un índice de las pobres expectativas que se tienen sobre el futuro de dicha persona en la tarea, mientras que si se atribuye el resultado negativo al bajo nivel de esfuerzo, se estará pensando que si de nuevo lo intentara y se esforzara, podría superarlo.

Los resultados obtenidos en el estudio ponen de manifiesto que el observador hace diferentes atribuciones sobre el éxito o el fracaso de un trabajador, en función del grado de poder que en él/ ella percibe. De forma parecida, Overbeck et al. (2006) pusieron de manifiesto que las personas tienden a ver a los trabajadores con poco poder como "motivados situacionalmente" mientras que los trabajadores que ocupaban cargos de alto poder se percibían como "motivados disposicionalmente" Según las autoras, la gente suele pensar que las personas poderosas se desenvuelven normalmente en situaciones libres de limitaciones en las que ellos deciden qué quieren hacer y de qué manera. Por el contrario, las personas con bajo poder se perciben como personas subordinadas a otras con más poder, que son quiénes realmente controlan sus acciones. Así, como ejemplo se puede pensar en la distinta explicación que los observadores dan sobre el he- cho de que un trabajador acuda a su oficina a trabajar en un día festivo. Mientras que si se trata de un directivo de la empresa, las personas atribuyen dicho comportamiento a la voluntad del directivo, si se trata de un empleado base, la gente atribuye la misma conducta a las órdenes de las personas situadas en lugares más altos de la jerarquía. Para el estudio se decidió analizar el patrón atributivo del observador ante un éxito y un fracaso obtenido por la persona que tiene o no poder en la empresa. Además, a diferencia del estudio de Overbeck, el objetivo no es analizar las diferencias entre atribuciones disposicionales vs. Situacionales, sino que se intentó delimitar la importancia de la controlabilidad, por lo que las causas presentadas fueron siempre internas diferenciándose en el grado de control de las mismas. Así, encontramos evidencia empírica acerca de la superioridad de las atribuciones controlables, cuando se debe explicar tanto el éxito como el fracaso de la persona poderosa. Este dato está en consonancia con los resultados de la investigación de Overveck et al. (2006) anteriormente mencionada, ya que de nuevo, se percibe a las personas que ocupan puestos de alto poder como personas motivadas disposicionalmente. Así, si estas personas triunfan en sus puestos de trabajo y consiguen beneficios para su empresa, el observador piensa que la causa que mejor explica estos resultados positivos debe ser una causa que esté bajo el control del líder, como es su nivel de implicación en la tarea. De la misma manera que si fracasa y no consigue los objetivos previstos, nadie parece cuestionar su habilidad, pero si se pone en duda su esfuerzo.

Por otra parte, llama la atención que el análisis de las atribuciones que el perceptor hace sobre el resultado obtenido por una persona que ocupa un puesto de bajo poder, ofrece un patrón atributivo distinto. En el caso de un fracaso de un subordinado, los perceptores tienden a explicar dicho resultado negativo haciendo uso de atribuciones incontrolables internas, esto es, refiriéndose a la poca habilidad que el sujeto tiene para realizar sus tareas. Así, se recurre a explicaciones que mencionan puntos débiles o limitaciones de las personas no poderosas cuando hay que explicar 
la no-consecución de objetivos. Por el contrario, el éxito de las personas con bajo poder se atribuye a causas controlables e internas del actor, como el esfuerzo. Dicho de otro modo, si una persona poco poderosa consigue un éxito, se piensa que se debe al gran esfuerzo que dicho sujeto ha tenido que realizar para conseguir alcanzar su meta, debido a los múltiples hándicaps a los que debe enfrentarse. Siguiendo la propuesta de Fiske et al. (2002), las personas que tienen bajo poder se perciben como poco competentes, lo que podría explicar que el observador atribuya los éxitos que consiguen estas personas al esfuerzo que realizan dado que no destacan por su capacidad y competencia.

De forma parecida a la propuesta, Iatridis y Fousiani (2009) realizan una investigación en la que se analiza el impacto del estatus socioeconómico de las personas en las atribuciones que el observador hace sobre el éxito o fracaso de las mismas. De forma concreta, plantean un escenario en el que presentan a una chica que finaliza sus estudios y realiza un examen para poder acceder a estudios de postgrado. En este caso se manipulaba tanto el estatus socioeconómico de la actriz (alto vs. bajo), como el resultado obtenido en una prueba para acceder a los estudios deseados (alto vs. bajo). Los resultados ponen de manifiesto que tanto en la condición en la que la actriz es de alto estatus y consigue aprobar, como cuando es de bajo estatus y suspende, los participantes realizaron más atribuciones a la habilidad de la protagonista del escenario. Sin embargo, cuando la chica era de alto estatus y fracasaba o cuando era de bajo estatus y aprobaba, el resultados se atribuía en mayor medida al esfuerzo.

A pesar de las coincidencias entre el presente estudio y el de Iatridis et al. (2009), se considera importante hacer notar que las particularidades del contexto en el que se hacen salientes el estatus y el poder que ostentan los protagonistas, son cruciales si el objetivo es analizar su impacto sobre las atribuciones. Así, se considera que en el caso del estudio de Iatridis et al., el hecho de centrarse en una estudiante con estatus socioeconómico alto hace que el perceptor piense que la posición social que ocupa no se debe a ella misma, sino la que le viene dada por su familia. Por el contrario, como ocurre en el presente estudio, cuando se presenta una persona que ocupa un puesto de alto poder en un contexto laboral, es más probable pensar que esta persona ha tenido que formarse y luchar para llegar a ocupar dicho puesto. Debido a estas diferencias, se piensa que los distintos patrones encontrados en ambos estudios quedan justificados, ya que en nuestro caso nos centramos en el ámbito laboral, en el que el estatus y el poder que poseen los individuos se confiere por el cargo que ocupan; mientras que en el estudio anteriormente citado, el estatus de la chica le viene dado por la posición socioeconómica de su familia.

Para concluir, se cree necesario investigar los efectos del poder en contextos reales en los que se pueda a acceder a poblaciones no universitarias, como por ejemplo en el ámbito empresarial o en organizaciones, en las que se puedan observar las interacciones entre supervisores y subordinados, y las explicaciones que ambos grupos dan ante distintos eventos que les ocurren dentro de su área de trabajo. Además, se considera importante seguir profundizando en el análisis de las relaciones entre variables socioestructurales como poder, estatus o su legitimidad, y el proceso atributivo. Futuros estudios deberían analizar estas relaciones no sólo desde el punto de vista del observador, sino también desde la perspectiva del actor. En este sentido, se considera interesante analizar la relación entre la dimensión controlabilidad y los resultados que obtienen las personas en su lugar de trabajo. Desde el punto de vista de las autoras, las atribuciones controlables o incontrolables pueden ser una herramienta útil tanto para actores como para observadores en el ámbito organizacional, ya que puede ayudar a conocer las distintas expectativas que las personas poseen sobre su futuro laboral y sobre el de las personas que le rodean.

\section{Referencias}

Anderson, C. \& Galisnky, D. (2006). Power, optimism, and risk-taking. European Journal of Social Psychology, 36, 511-536. 
Buchanan, G. \& Seligman, M. (1985). Explanatory style. Hillsdale, NJ: Lawrence Erbaum.

Dahl, R. (1957). The Concept of Power. Behavioral Science, 2, 201-215.

Darley, J. M. \& Gross, P. H. (1983). Hypothesis-confirming bias in labelling effects. Journal of Personality and Social Psychology, 44(1), 20-33.

Ellemers, N. (1993). The influence of socio-structural variables on identity management strategies. En W. Stroebe \& M. Hewstone (Eds.), European Review of Social Psychology, 4 (p.p. 27-58). Chinchester, UK: Wiley.

Ellemers, N., Spears, R. \& Doosje, B. (1997). Sticking Together or Falling Apart: In-Group Identification as a Psychological Determinant of Group Commitment Versus Individual Mobility. Journal of Personality and Social Psychology, 72, 617-626. doi: 10.1037/0022-3514.72.3.617.

Ellemers, N., Van Knippenberg, A. \& Wilke, H. (1990). The influence of permeability of group boundaries and stability of group status on strategies of individual mobility and social change. British Journal of Social Psychology, 29, 233-246.

Fincham, F. \& Bradbury, T. (1992). Assessing attributions in marriage: The Relationship Attribution Measure. Journal of Personality and Social Psycho$\log y, 62(3), 457-468$.

Fiske, S. T. (1993). Controlling other people: The impact of power on stereotyping. American Psychologist, 48, 621-628.

Fiske, S. T., Cuddy, A. J. C., Glick, P. \& Xu, J. (2002). A model of (often mixed) sterotype content: Competence and warmth respectively follow from perceived status and competition. Journal of Personality and Social Psychology, 82, 878-902.

Fiske, S. T. \& Depret, E. (1996). Control, interdependence and power: Understanding social cognition in its social context. European Review of Social Psychology, 7, 31-61.

Georgesen, J. C. \& Harris, M. J. (1988). Why's my boss always holding me down? A meta-analysis of power effects on performance evaluations. Personality and Social Psychology Review, 2, 184-195.

Gilbert, D. \& Malone, P. (1995). The correspondence bias. Psychological Bulletin, 117 (1), 21-38.
Guinote, A. (2007). Power and Goal Pursuit. Personality and Social Psychology Bulletin, 33(8), 1076-1087.

Huston, T. L. (1983). Power. En H. H. Kelley et al. (Eds.), Close relationships (pp. 169-219). Nueva York: Freeman.

Iatridis, T., \& Fousiani, K. (2009). Effects of status and outcome on attributions and just-world beliefs: How the social distribution of success and failure may be rationalized. Journal of Experimental Social Psychology, 45, 415-420

Jones, E. E. \& Pittman, T. S. (1982). Toward a general theory of strategic self-presentation. In J. Suls (Ed.), Psychological perspectives on the self, 1 (p.p. 231-262). Hillsdale, NJ: Lawrence Erlbaum.

Keltner, D. Gruenfeld, D. H. \& Anderson, C. (2003). Power, approach and inhibition. Psychological Review, 110, 265-284.

King, L. A. (1995). Wishes, motives, goals, and personal memories: Relations of measures of human motivation. Journal of Personality, 63, 985-1007.

Kipnis, D. (1976). The powerholders. Chicago: The University of Chicago Press.

Lee, F., Peterson, C. \& Tiedens, L. Z. (2004). Mea culpa: Predicting stock prices from organizational attributions. Personality and Social Psychology Bulletin, 30, 1636-1649.

Levinger, G. (1959). The development of perceptions and behavior in newly formed social power relationships. En D. Carwright (Ed.), Studies in social power (pp. 83-98). Ann Arbor, Ml: Institute for Social Research.

Lippit, R., Polansky, N. \& Rosen, S. (1952). The dynamics of power: A field study of social influence in groups of children. Human Relations, 5, 37-64.

Overbeck, J., Tiedens, L. Z. \& Brion, S. (2006). The powerful want to, the powerless have to: Perceived constraint moderates causal attributions. European Journal of Social Psychology, 36, 479-496.

Peterson, C. (2000a). Optimistic explanatory style and health. En J. Gillham (Ed.), The science of optimism and hope: Research essays in honour of Martin E. P. Seligman (pp. 145-161). Philadelphia: Templenton Foundantion Press.

Peterson, C. \& Seligman, M. E. P. (1984). Causal explanations as a risk factor for depression: Theory and evidence. Psychological Review, 91, 247-374. 
Rodríguez-Bailón, R., Moya, M. \& Yzerbyt, V. (2000). Why do superiors attend to negative stereotypic information about their subordinates? Effects of power legitimacy on social perception. European Journal of Social Psychology, 30, 651-671.

Steensma, H., Den Hartigh, E. \& Lucardie, E. (1994). Social categories, just world belief, locus of control and causal attributions of occupatonal accidents. Social Justice Research, 7(3), 281-299.

Tiedens, L. Z., Ellsworth, P. C. \& Mesquita, B. (2000). Sentimental stereotypes: Emotional expectations for high and low status group members. Personality and Social Psychology Bulletin, 26(5), 560-575.
Trope, Y. (1986). Identification and inferential processes in dispositional attribution. Psychological Review, 91, 239-257.

Turner, J. C. (1991). Social Influence. Buckingham: Open University Press \& Pacific Grove, Brooks/Cole.

Weiner, B. (1985). An attibutional theory of motivation and emotion. Psychological Review, 92, 548-573.

Weiner, B. (1986). An attributional theory of motivation and emotion. New York: Springer-Verlag.

Winter, D. G. (1973). The power motive. New York: Free Press. 